\title{
Routing Algorithm for Heterogeneous Wireless Network in Vanet
}

\author{
Ravi Shankar Shukla ${ }^{1}$ and Neeraj Tyagi ${ }^{2}$ \\ ${ }^{I}$ Department of CSE \& IT, Invertis University, Bareilly, India and Research Scholar Dept. of CSE, MNNIT, \\ Allahabad, India \\ ${ }^{2}$ Department of Computer Science \& Engineering, MNNIT, Allahabad, India
}

\begin{abstract}
Today, Vehicular Ad Hoc Network (VANET) is an emerging technology. The routing problems in heterogeneous network become popular research area in the field of VANET for supporting different aspects of Intelligent Transportation System (ITS) applications. With the progress of wireless radio technology, telecommunication, various wireless specifications and protocols form the heterogeneous network. In this paper, we integrate cellular ( $3 G)$ network and Vehicular Ad-Hoc Network (VANET) into a hybrid network. This hybrid network is called heterogeneous wireless network $(H W N)$ with multi-cells design to overcome the limitation of cellular network and Ad-Hoc network. We also, propose a routing algorithm for HWN. The simulation results show that HWN with the proposed routing algorithm has minimum request block rate and transmission time.
\end{abstract}

Keywords: Heterogeneous Wireless Network, VANET, Routing Algorithm

\section{Introduction}

In current years, the progress of wireless communication technology brings convenience to our practice. In addition, a variety of wireless personal communication systems have been deployed globally across different geographical location so that; mobile users can communicate with others and access the public networks anytime and anywhere. Therefore, wireless network must provide everywhere communication capability and information access apart from of user's position.

There are many wireless plans deployed according to the necessities and the characteristics of networks. Most of them can be classified according to their transmission rate and coverage range. A satellite system provides worldwide coverage with low transmission rate. The Wireless Local Area Network (WLAN) manage at Mbps rate (normally $2 \mathrm{Mb} / \mathrm{s}$ ) more than a range of hundreds meters (normally $>=150$ meter using IEEE $802.11 \mathrm{p}$ protocol). Also, the popular $3 \mathrm{G}$ system has the coverage in the range of more than a few kilometers with the transmission rate of about $200 \mathrm{~kb} / \mathrm{s}$ or higher.

Moreover, the Vehicular Ad-Hoc Network (VANET) is a different wireless network not including communication infrastructure. It can be characterized by dynamic topology with vehicular node mobility and narrow bandwidth. Each Vehicular Node (VN) operates not only as a host but also a router. It forwards the packet to other nodes using peer-to-peer communication. A lot of work has been discussed about efficiently finding the multi-hop routing paths $[3,4]$ and a number of resource factors that require to be considered in uniform environment are discussed in [5,6]. These routing algorithms can be mostly classified into two categories:

(a) One is proactive protocol, in which all VNs proactively keep the information of their neighbors in information table. The information stored in the table of VNs includes number of hopes, TTL, transmit rate, etc. When some VN start route request message, this protocol can be aware of the adjacent VNs' state without any extra discovery and delay. Hence, adjacent VNs must periodically exchange the information to get the timely bring up to date of their information table. The period of updating-table time $T$ in proactive routing protocol relies on the routing table update mechanism. When $T$ is low, outdated routing table information in $\mathrm{VN}$ is unavoidable. When $T$ is high, there will be needless overhead of updating routing table. Moreover, not all of VNs deliver and receive data in each moment in time. Therefore, such protocol is not included in the proposed algorithm.

(b) The other category is reactive protocol, which couples with the on-demand nature. This protocol eliminates the need of updating table information among adjacent VNs. When a VN uses this protocol, it takes some time during the process of route discovery. The proposed routing algorithm in this paper uses reactive routing protocol as it eliminates the need of updating table information so that unnecessary use of bandwidth can be avoided. 


\section{Related Work}

Bearing in mind a range of features and restrictions of wireless communication technology, the VANET research works are still simulation based but there are some quite exciting and upcoming projects expected to be available before long in the real world situations. The scope of this work is associated with the coverage of complete communication and routing paradigm in vehicular ad hoc networks. Initially, the simulation schemes would be considering the generic mobility patterns of the road networks. Therefore, the study highlighted here is also depending upon various rigorous simulation scenarios. A range of heterogeneous wireless networks are formed, but most of them are not implementable. Presently, many researchers are working on the mission of $4 \mathrm{G}$ also known as Beyond Third generation (B3G). The main aim of $4 \mathrm{G}$ mission is to put together all kind of heterogeneous networks [9]. On the other hand, the mission poses many research challenges and issues, such as handoff and location management [8]. After that, the incorporation of the popular 3G cellular network and vehicular Ad-Hoc network to form Heterogeneous Wireless Network (HWN) design is introduced in this paper. The $4 \mathrm{G}$ is a mission integrating all types of the heterogeneous wireless network. At present is a lot of work accepted to develop the routing protocol and algorithm in HWN toward 4G [14]. However, most of the work only center on the theoretical network architecture of $4 \mathrm{G}$ without details or just consider only base station (BS) assumption without considering the congestion issues [13]. Moreover, these researches only discussed about the routing algorithm of original cellular network or original Ad-Hoc network. In this paper, we propose heterogeneous wireless network (HWN) which is the hybrid network of popular 3G cellular network and Vehicular Ad-Hoc network. It takes the advantages of the major $3 \mathrm{G}$ system and the transmission rate in Vehicular Ad-Hoc network. The proposed HWN can reduce the block rate of the Public networks access and support larger coverage of Vehicular Ad-Hoc network. We also introduce a new routing algorithm for HWN without extra requirements.

\section{Proposed Heterogeneous Wireless Network Architecture}

The proposed heterogeneous wireless network, integrates the characteristic of the cellular network (e.g. 3G system) and Vehicular Ad-Hoc network as shown in Figure 1. It is assumed that each Vehicular Node (VN) equips with the Mobile Router (MR) and cellular interface and Ad-Hoc interface. Therefore, the routing protocol can make a choice of selecting the correct mode or interface to deliver the packets. In order to form HWN environment, we distribute the BS along road side. We also assume that the coverage of each BS is $1000 * 1000$ square meters. It is also assumed that, it is an IP environment in which each VN and BS has unique IP as ID.

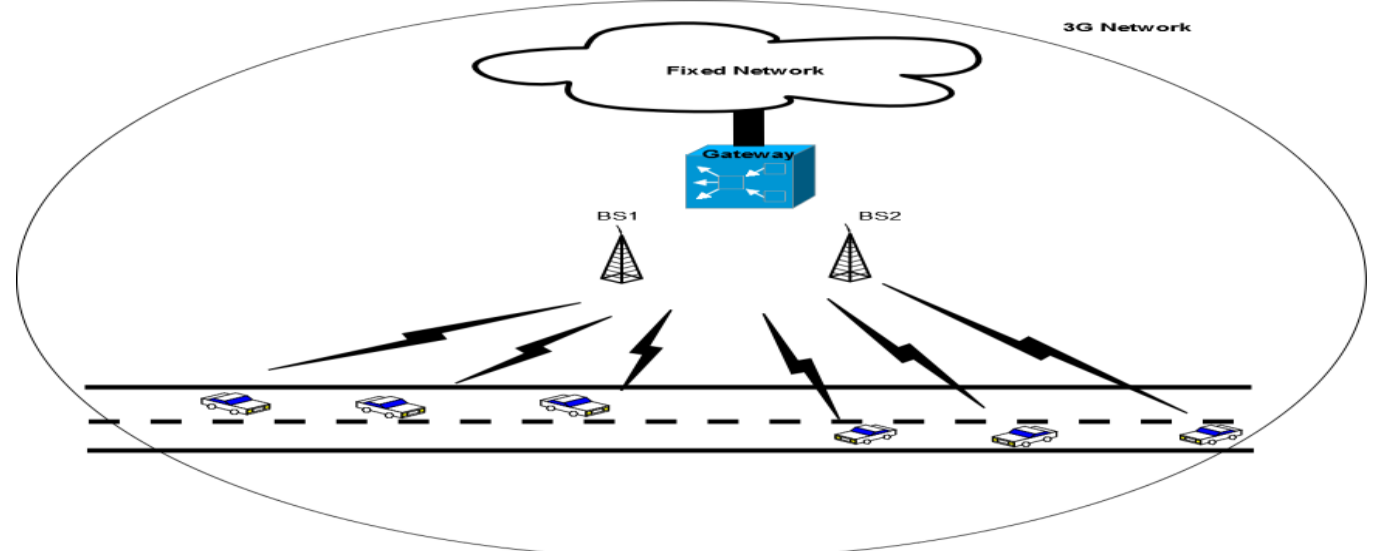

Figure1. Proposed heterogeneous wireless network architecture for VANETs

The HWN design that we proposed is used for the vehicular applications. There are many factors in vehicular network applications such as availability, reliability, and throughput that needed to improve the routing algorithm performance for forwarding data.

To improve the routing algorithm performance, we resolve two issues rose in the existing HWN.

(i) The disadvantage of existing HWN is that it only supports the data transmission between the specific source and destination. When specific source node has many routing paths to send data packets to destination, and useful routing path is selected.

(ii) Due to the multi-cells design of HWN there may be data traffic load in HWN is a useful scheme of distributing the data traffic is required to enhance the total data throughput and provide better services 
to number of vehicular nodes.

The proposed routing algorithm solves these two issues that we discussed later in this paper. Now, we introduce the architecture of our proposed HWN. The HWN is composed of Base Station (BS), vehicular node that equips with Mobile Router (MR), 3G network and gateways. The design of proposed HWN is shown in Figure 1. Vehicular node can access the public network network through different cellular cells or communicate with other vehicular nodes directly or via Base Station (BS). Hence the vehicular device in HWN can have different behavior in the homogeneous network. To understand the different behavior of HWN is shown in Figure 2.

In Figure 2 there are two types of communication possible for vehicular network

(a) Communication between specific source to specific destination

The communication between specific source to destination can further divided into three sub-categories:

(i) Source and destination are in under in same BS

If a source (Source1) wants to deliver data packets to destination (Destination1), Source1 deliver the packets to Destination1 by multi-hop routing through intermediate nodes without any assistance from the cellular network (Source1 can deliver packets to Destination1 by using its own Ad-Hoc network). The arrow marked (1) in Figure 2 shows this communication scenario.

(ii) Source and destination are in under different BS, but form a Ad-hoc region

If Source2 and Destination2 are in different cells (BS) but sufficiently close to each other so as to form their AdHoc region, then Source2 can deliver packets to Destination2 through their Ad-Hoc network without using the of the Base Station (BS). The arrow marked (2) in Figure 2 shows this type of communication.

(iii) Source and destination are in under different $B S$

When Source3 under BS1 wants to deliver packets to Destination3 which is under BS2 then, Source3 will use the cellular network to deliver data packets. The Source3 will first send the data packets to the BS1 (as shown by arrow marked (3)). Then, packets are subsequently by BS1 (arrow marked (3)) delivered through the fixed network to BS2. Finally, BS2 will deliver the data packets to Destination3 (arrow marked (3) in Figure 2).

(b) Communication between specific source to Public Networks

In addition, VN can connect to the Public network through the cellular network to access public networks applications rather than making any communication to other VNs.

The proposed HWN incorporates both types of communication possibilities explained above. The detail of these communication categories are explained in next section.

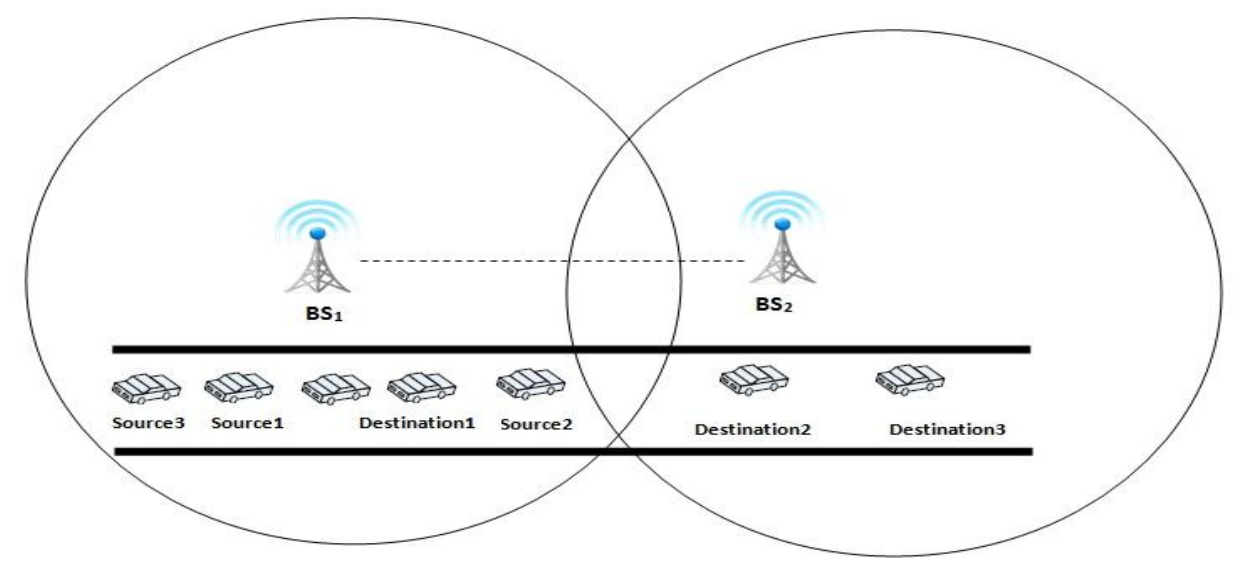

Fig2. Various Source \& Destinations in heterogeneous wireless network architecture

\section{Proposed Road Based Routing Algorithm}

The integration of VANET and cellular network is used to form the heterogeneous wireless network. In this section a new routing algorithm, named Road Based Routing Algorithm (RBRA) is proposed for our heterogeneous wireless network. The two major processes of the RBRA are Route Discovery process and Distribution Process.

The flowchart of RBRA (Road Based Routing Algorithm) is shown in Figure. 3. We also consider and describe the network interface used by $\mathrm{VN}$ as mentioned. We already classify the requirements of traffic load into two parts: (i) the routing path from the specific source and destination traffic and, (ii) the routing path from VN to Public networks. The difference between these two traffic loads is the destination of the request. The destination can be VN or the server connected to Public network by fixed network. 
If the application of VN can decide the requirement of the Public network access, the VN can send the Route Request message (RREQ) messages by using cellular interface to the cell (BS) in which it is registered, without flooding the RREQ messages. However, if the application of VN is not able to decide the type of data traffic for the Route Request message (RREQ), it can send the RREQ message to its BS. BS can check the destination in database table which includes visited vehicle or own location vehicle registered under the BS.

- If the check successes, the specific source and destination traffic is chosen.

- Otherwise, the destination is the server connected to fixed network. Thus VN must transmit data to destination through BS and fixed network. We call such kind of data traffic as "Public networks Access", and the public network access traffic is chosen.

\section{(i) Routing path for specific source and destination}

If $\mathrm{VN}$ initiate the routing path to specific destination in HWN, the Road Based Routing Discovery process (RBRDP) starts to find the specific destination by using Ad-Hoc and cellular interface. If a reachable routing path is found, the proposed routing algorithm evaluates the route metric for each reachable route and selects one routing path with minimum route metric to transmit data. If no such reachable path exists, then the request is blocked.

\section{(ii) Routing path for Public networks Access}

When VN wants to access the public networks, it connects itself to the registered cell by using cellular interface. Then, BS runs the bandwidth capacity test to check whether it has enough bandwidth to this request.

If the capacity test is succeeded, then the BS sends ACK message back to VN. The ACK message indicates that the bandwidth is reserved by BS for requesting VN. Next, the VN establishes the routing path to BS for data transmission. Finally, the VN accesses the Public network through fixed network via BS.

If the capacity test fails, the BS starts a Load Distribution Process to find a reachable routing path from the neighboring BS. VN receives a Route Acknowledgement (RACK) message from registered BS. The RACK message indicates to requesting $\mathrm{VN}$ that registered BS does not have sufficient bandwidth to access public network. The RACK message sent by BS also includes cell IDs of its neighboring BS. Thus, VN triggers Road Based Route Discovery process (RBRDP) for specific available cells ID's sent by its BS. Now, VN can use a ad-hoc and cellular interface to flood RREQ message to find the destination by using communication between specific source and destination in heterogeneous wireless network. If VN find a reachable path within a limited time, than it evaluates the route metric for the reachable path. Next, the VN select the path with the minimum route metric to forward the data. If $\mathrm{VN}$ does not find any reachable path within a limited time, the route request (RREQ) message is blocked. In next section, we will describe the RBRDP and LDP processes in detail.

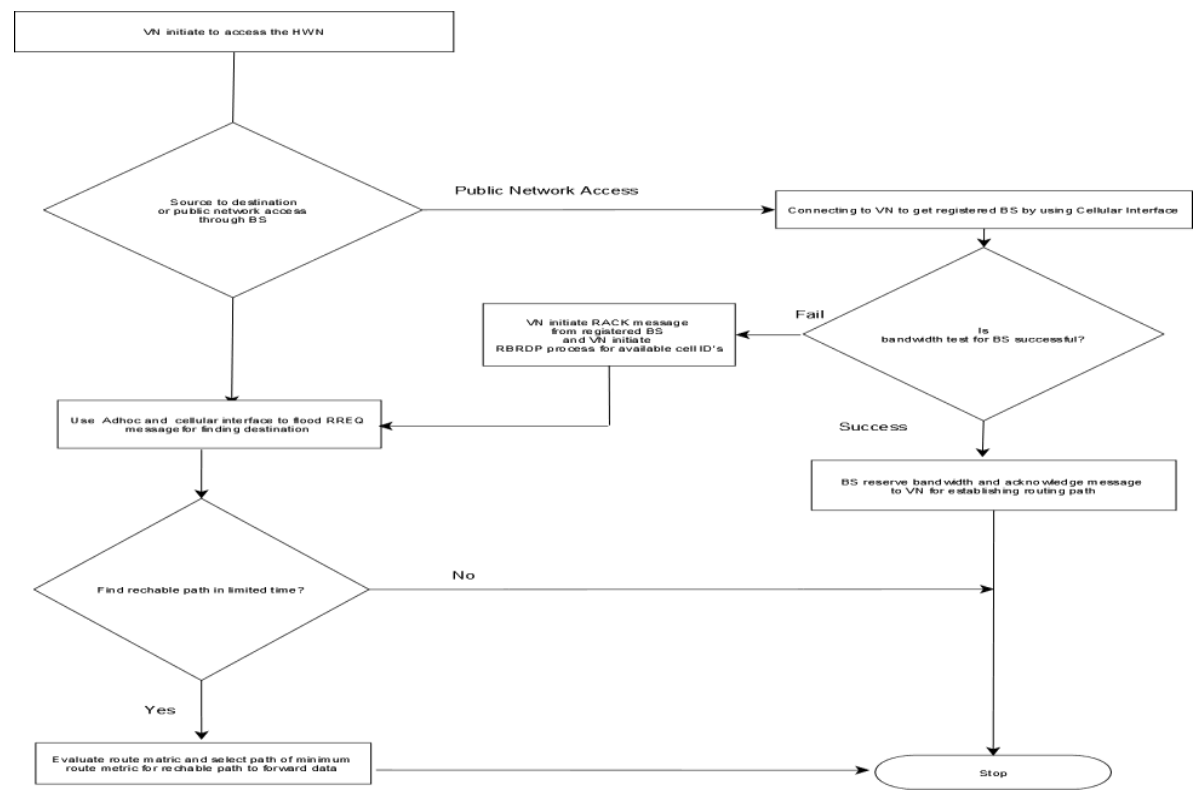

Fig.3. Flowchart Road Based Routing Algorithm in Heterogeneous Wireless Network 


\section{Road Based Routing discovery process}

Based on proposed HWN mentioned earlier, the proposed routing algorithm is used to find out the path to send or deliver data packets in the network. We have already discussed that our proposed routing algorithm is based on reactive routing protocol.

It is not necessary that every VN require accessing the cellular interface (i.e BS). But some of the VNs that do not access the cellular interface also have the ability to forward or deliver data packets to other VNs through the Ad-hoc region they formed. VNs that really require accessing the cellular interface can send the Route Request Messages (RREQ) to BS. During route discovery process, the RREQ message will be broadcasted to all the VNs that are in the Ad-Hoc region of initiating VN as well as to the BS in which initiating $\mathrm{VN}$ is registered. The RREQ message received by VNs and BS can also broadcast the same RREQ message to their neighboring VNs and BS. This broadcasting continues until destination node is found. This continues broadcasting in Route Discovery process increases the Route Reply Packet Overhead.

However, if we limit the number of the forward hops in our routing protocol, the on-demand flooding RREQ message overhead may be reduced. The TTL (Time to Live) in the RREQ message also limits the delay time of whole routing process. The Road Based Routing algorithm (RBRA) uses reactive routing which is more suitable and better fit in HWN. .

\section{(a) Route Discovery Phase}

Our RBRDP (road based routing discovery process) is designed for heterogeneous wireless network and it is the modification of the reactive DSR (Dynamic Source Routing) routing protocol which is designed for homogeneous network. In routing discovery phase, we assume that BS (Base Station) will act as a VN during routing discovery process. The bandwidth usage, delay of transmission and the mobility of every VN may be different, but every VN must follow the road topology and should be single administrative domain. However, the transmission range and frequency band is same for all VNs as it follow the IEEE $802.11 \mathrm{p}$ specification. Similarly, the transmission range, and frequency band is same for all BS as it follow the cellular standards. But, all BS differs in bandwidth usage and delay of transmission. We already assumed that each node (VN or BS) has a unique IP, then, the "Source ID" field and "Destination ID" field in RREQ message can be filled with specific IPs.

The RREQ message format and their field are shown in Fig. 4.

\begin{tabular}{|l|l|l|l|l|l|}
\hline Source ID & Request ID & $\begin{array}{l}\text { Destination } \\
\text { ID }\end{array}$ & Time to live & $\begin{array}{l}\text { Absolute } \\
\text { Route }\end{array}$ & $\begin{array}{l}\text { Request } \\
\text { Bandwidth }\end{array}$ \\
\hline
\end{tabular}

Fig.4. The RREQ Message Format

When some VNs initiate to access the HWN (i.e. cellular interface or Ad-Hoc region), then BS as well as vehicular node (VN) participate in the routing discovery process. Therefore, when node (VN or BS) receives the RREQ message, it checks all the route field of RREQ to determine that whether all fields contain the absolute information. If the incomplete routing path exists in routing table of node, then node will discard the RREQ message. Otherwise, it re-floods the packet to reachable neighbors and appends the host id as destination in the absolute route field. The process of re-flooding of packets continues until destination is found. If the destination is reachable, the RREP message contains the absolute routing path information toward the source. Hence, the routing path is established.

Through the use of BS, the original source and unreachable destination pair in heterogeneous Ad-Hoc network can find alternate path to deliver packets. If there exists an alternate path, then the request block rate can be reduced. The request block rate is defined:

$$
\text { Block rate }=\frac{\text { Number of request blocked to access the network }}{\text { total number of request to access the network }}
$$

When BS receives the RREQ message, it can check the visitor database table to find the reachable destination (VN) to avoid re-flooding of RREQ message. If the destination VN is found in its visitor database table or home database table, then it can send the RREQ message to the reachable VN. Otherwise it forwards the RREQ message to other BS through cellular network. The same process continues till destination is found.

The modified routing discovery phase is shown in Fig. 5(a) \& (b). The source node can be any VN in the HWN. When the source node initiates the route request, it checks the route cache. If there is a route in route cache that contains the destination ID, the destination is reachable. Then, the source node copies the routing path in the packet header and forwards the packet to next-hop node. If the route in route cache does not exist, the source node buffers the forwarded data and floods RREQ messages to reachable neighboring nodes until 
receiving the RREP (Route Request Reply) messages in a limited time.

Here, we assume that the requests initiated by the source node have queuing property. The request of data transmission that is initiated earlier by the source node is processed first. The intermediate node can be either a VN or BS.

(a) When the intermediate node (VN) receives the RREQ message, it checks the "TTL" fields in RREQ message to decide whether the message will be discarded or not. If the incomplete route is recorded in absolute route field then the host discards the RREQ message. When the intermediate node that receives the duplicate RREQ message from the same source ID, then it discards the RREQ message to minimize Route Request Packet Overhead. Thus the traffic of flooding RREQ message received by intermediate node can be removed if they are originated from of the same source ID. If the host ID is same as the destination node for the request, the host sends the RREP message backward to the source node. Otherwise, the VN appends host id and available bandwidth in absolute route field in RREQ message and forward to reachable next-hop by ad-hoc and cellular interface. When the intermediate node receives the RREP message, it updates its available bandwidth and forwards the RREP message to next-hop recorded in the RREP message.

(b) If the intermediate node is BS, it checks:

(i) The visitor database is used to find the accessible VNs in its record as well as it also checks the bandwidth request to serve the RREQ initiated by source. If the destination is reachable, then BS updates a host ID and available bandwidth in the source ID and bandwidth request field respectively in the RREQ message. The updated RREQ message is then sent to destination VNs.

(ii) The home database table is used to find whether the VN is registered in it or not. If VN is registered in its database table, then it sends the RREQ message to reachable destination VN. For sending the message, it appends the host ID and available bandwidth in absolute route field in RREQ message. Otherwise, BS check the neighboring BS to forward the RREQ message through fixed network to find the destination node, such as Source3-BS1-BS2-Destination3 path shown in Figure 2.

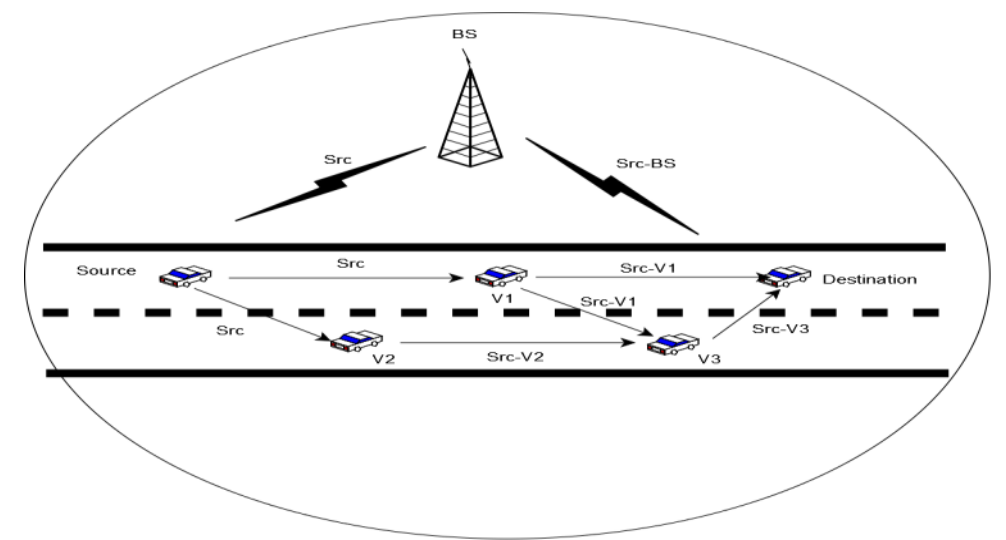

Figure (a)

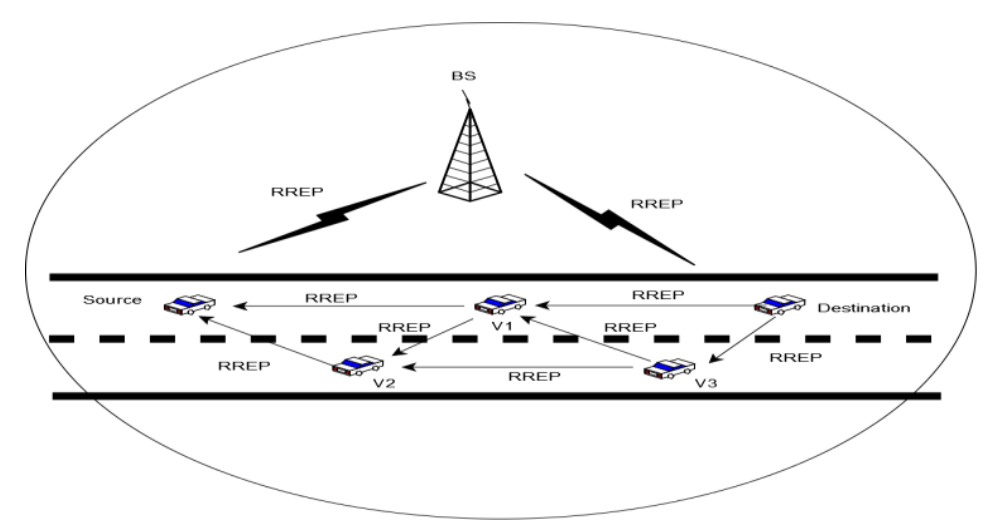

Figure (b)

Figure 5. Route Discovery steps 
The pseudo code for Road Based Routing Algorithm is shown as.

Pre-condition: Vehicular node initiate request to access HWN

Post-condition: A minimum route metric path established

\section{$\underline{\text { Road Based Routing Algorithm }}$}

1. VN initiate route request (r) to access HWN

2. if $\mathrm{r}$ is for public networks access then

$\mathrm{VN}$ connect to its registered BS using cellular interface

if capacity test of BS succeeded then

$\mathrm{BS}$ reserves the bandwidth for $\mathrm{VN}$

networks

BS sends an ACK message to VN and established the routing path from $\mathrm{VN}$ to public

Else // find another alternative routing path

BS starts a Load Distribution Process (LDP)

BS checks another neighboring BS that can fulfill the incoming request

BS fills the neighboring BS ID in RACK message

RACK message in send to source node (VN)

VN initiate route request (r) to access public network through this neighboring BS ID

VN floods PREQ message for finding alternate path $\left(\mathrm{r}_{1}\right)$

If $r_{1}$ is found in limited time then

Evaluate route metric for reachable path

Select the path with minimum route metric to forward data

Else

Write ("No path exits for public networks access");

3. if $r$ is for specific source to destination then

//route discovery phase

VN floods RREQ message for finding path $r$

If $\mathrm{r}$ is founded in limited time then

//route selection phase

Evaluate route metric for reachable path

Select the path with minimum route metric to forward data

Else

Write ("No routing path exits between route and destination");

We select the route with higher average value of available to the bandwidth.

\section{(b) Load Distribution Process}

In this section, we describe the Load distribution in detail. The capacity of BS is usually limited. We formally describe the meaning of capacity test of BS. The capacity check of BS refers to the available bandwidth of BS to fulfill the incoming request. When capacity test of reachable BS is unsuccessful, this BS becomes the overcrowding. Once VN wants to access the Public networks through the overcrowding BS but the request is not fulfilled, then the load distribution process initiates. The objective of the process is to find out an additional accessible routing path to access the Public network through some other accessible BS as the registered BS of VN becomes overcrowding. The existing cellular network supports only their registered VNs. If the BS is overcrowded, then the request sent by their registered VNs could not process. We propose an expansion of cellular network that eliminates the above problem and also supports multi cells services through the Vehicular Ad-Hoc Network. In Figure 2 if the BS1 becomes overcrowding, the request initiated by Source 2 will access the network through the routing path Source2- BS2- Destination2. Consequently, the request of Source2 may perhaps access the network from being blocked by BS1 with the help of proposed scheme and the request block rate can be minimized.

\section{Simulation parameter and performance Analysis}

In this section, we present the performance evaluation of the proposed RBRA (Road Based Routing Algorithm) in HWN (Heterogeneous Wireless Network). The simulation model was based on the Network Simulation (NS2) and VANET. An unslotted carrier sense multiple access with collision avoidance (CSMA/CA) is used for data transmission in MAC layer. The distributed position of VNs in the HWN is the high way scenario and located. All VNs are registered to BS. The registered BS of VN is depended on the position of the VN. The handoff scheme of HWN is based on the "hard handoff". When VN moves to 
neighboring cells, it triggers the routing discovery process to establish the new route toward the new cell and it drops the previous connection immediately. Moreover, we give some definitions in HWN because that HWN must satisfy the Vehicular Ad-Hoc network and cellular network requirements.

Table 1. Simulation Parameter

\begin{tabular}{|c|c|}
\hline Parameters & Value \\
\hline Number of BS & 6 \\
\hline Coverage of BS & $1000 * 1000$ Square meters \\
\hline Wireless specification & IEEE 802.11p \\
\hline Maximum hops of each route & 8 \\
\hline Bandwidth range & Highway Scenario \\
\hline Mobility Model & 1 second \\
\hline Pausing time & $0-120 \mathrm{~km} / \mathrm{h}$ \\
\hline VN Speed &
\end{tabular}

\section{Performance Analysis}

The Figure 6 shows the design evaluation of Ad-Hoc network and proposed RBRA (Road Based Routing Algorithm). As the requirements in the uniform environment DSR don't engage the cellular network but the RBRA involve in both adhoc and cellular network. So, the request block rate of RBRA can be greatly reduced about 40 60\% compared with DSR in heterogeneous wireless network.

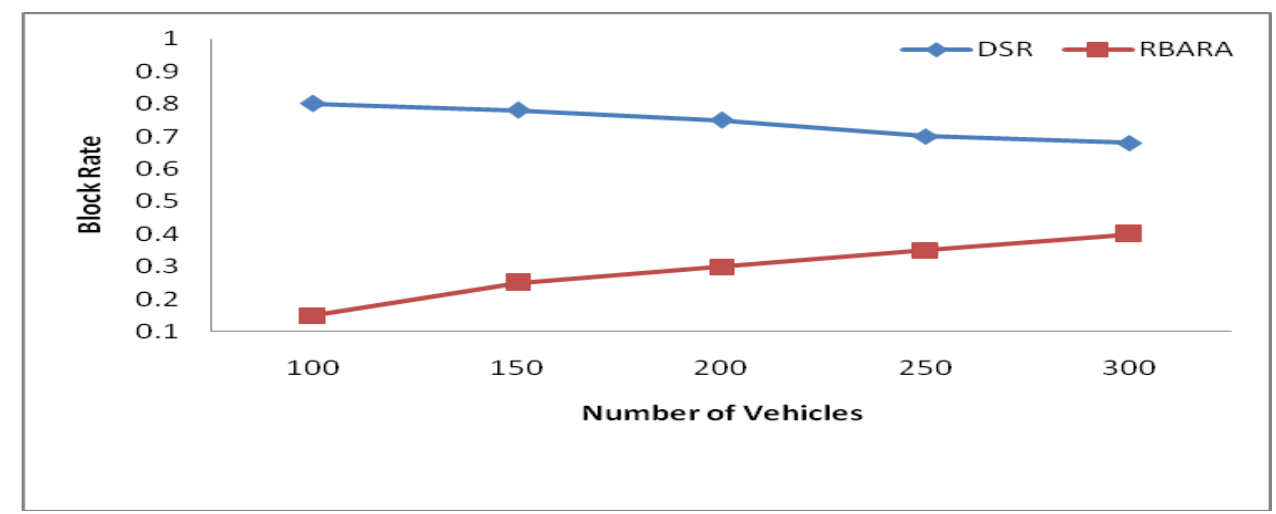

Fig. 6 Request block rate with DSR vs. Road Based Routing Algorithm in HWN

Figure 7 evaluate the transmission time between the DSR and proposed RBRA. The transmission time of the proposed RBRA is lesser than that of DSR. The amount of send data is set up to $2 \mathrm{Mb}$.

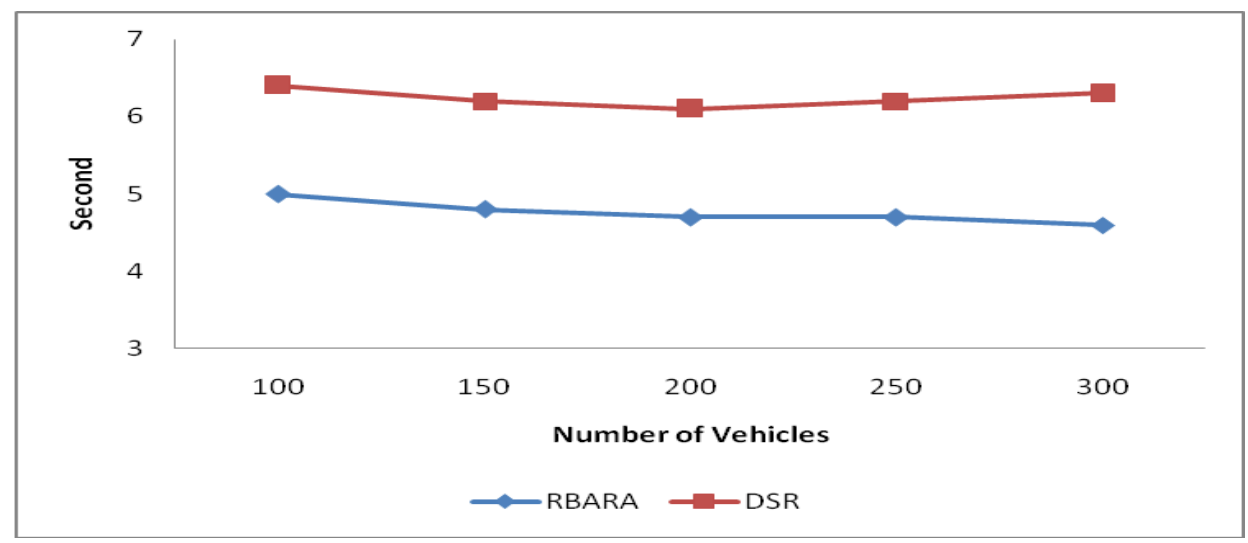

Figure. 7. Transmission Time Dynamic Source Routing vs. Road Based Routing Algorithm 
The evaluation of request block rate of RBRA in multi-cells situation with and without load distribution process is shown in figure 8 . The proposed RBRA minimizes the request rate. Therefore, we can provide services to more number of vehicular nodes to access the network with load distribution process.

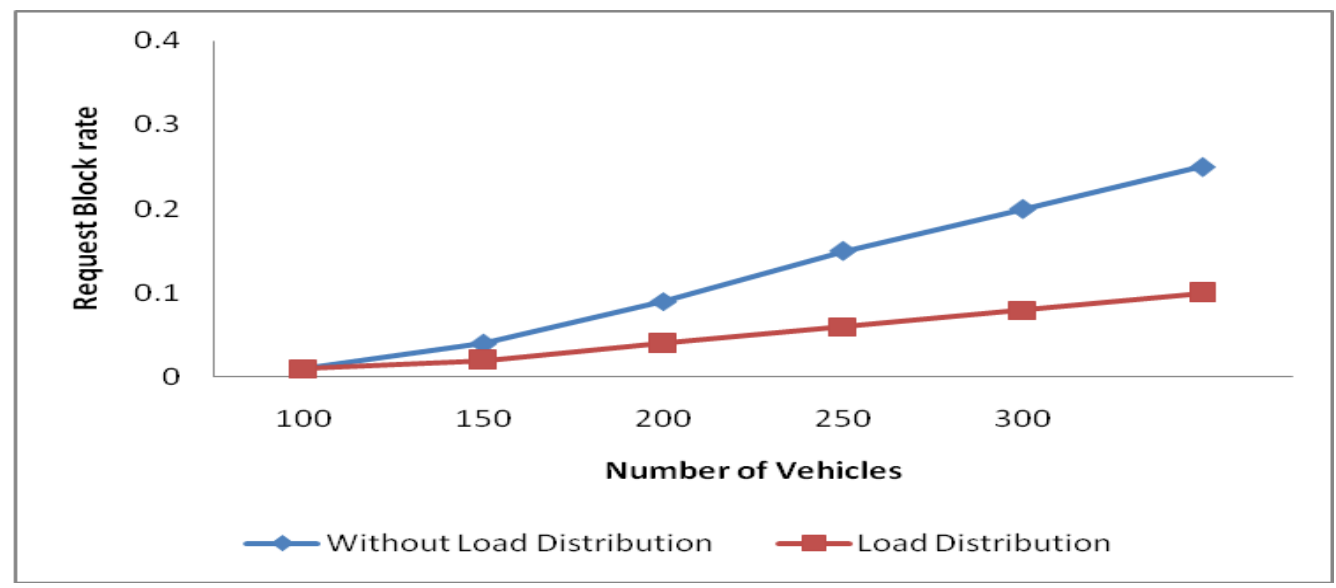

Figure 8. With and Without Load Distribution Process

\section{Conclusion}

In this paper, we have considered a heterogeneous wireless network consist of Vehicular Ad-Hoc network and cellular network with multi-cells. Taking the advantages of uniform network in heterogeneous wireless network, we have also proposed the RBRA (road based routing algorithm) which consists of two processes: road based routing discovery process and load distribution process. The heterogeneous wireless network design and the proposed routing algorithm can reduce block rate. Moreover, the load distribution process uses the simple scheme to check whether BS is congested or not.

\section{References}

[1] Himanshu Tyagi1, A. K. Vatsa1 (2011) "Seamless Handoff through Information Retrieval in VANET Using Mobile Agent" Vol.8.

[2] Muhammad Nawaz Khan,Ishtiaq Wahid \& Gulzar Mehmood (2012) "A Global Mobility Management Scheme for Reducing Overall Handover Latency in IP based VANETs" in IJASUC) Vol.3.

[3] X. Zhang, and L. Jacob, "Adapting Zone Routing Protocol for Heterogeneous Scenarios in Ad Hoc Networks", International conference on parallel processing (ICPP), Oct., 2003.

[4] G. R. Dattatreya, S. S. Kulkarni, H. Martinez, and R. Soto, “Adaptive control of heterogeneous ad hoc networks", IEEE International Conference on Systems, Man, and Cybernetics, Volume: 5, pp.3431 -3436, Oct., 2000.

[5] M. S. Jian, P.L. Wu, and C. N. Lee, "On-demand flow regulated routing for ad-hoc wireless network" IEEE Conference on Wireless Personal Multimedia Communications, vol.1, pp. 242-246, Oct., 2002

[6] M. S. Jian, C. C. Chen, and C. N. Lee, "Ad hoc On-Demand Resource Management using Improved Rank-based Fitness Assignment" , Proceeding On WPMC, pp.256-258, Oct., 2003.

[7] A. Bria, F. Gessler, O. Queseth, R. Stridh, M. Unbehaun, Wu. Jiang, J. Zander, and M. Flament, "4th-Generation Wireless Infrastructures: Scenarios and Research Challenges", IEEE conference on Personal Communications, vol.8, Dec., 2001

[8] U. Varshney, and R. Jain," Issues in emerging 4G wireless networks", IEEE Journal on Computer, vol.34, no.6, pp.94-96, Jun., 2001.

[9] W. Kellerer, C. Bettstetter, C. Schwingenschlogl, and P. Sties," (Auto) mobile communication in a heterogeneous and converged world", IEEE Journal on Personal Communications, vol.8, no.6, pp.41-47, Dec., 2001.

[10] V. Marques, R. L. Aguiar, C. Garcia, J. I. Moreno, C. Beaujean, E. Melin, and M. Liebsch, " An IP-based QoS architecture for 4G operator scenarios", IEEE Journal on Wireless Communications, vol.10, no.3,pp.54-62 , Jun., 2003.

[11] C.-H. Chou, K.-F. Ssu, and H. C. Jiau, "Geographic forwarding with dead-end reduction in mobile ad hoc networks," IEEE Trans. Veh. Technol.,vol. 57, no. 4, pp. 2375-2386, Jul. 2008.

[12] Reinaldo Bezerra Braga and Herve Martin (2011) "Understanding Geographic Routing in Vehicular Ad Hoc Network" in IARIA.

[13] R. Chang, W. Chen, and Y. Wen, "Hybrid wireless network protocols", IEEE Transactions on Vehicular Technology, vol.52, no.4, pp.1099-1109, Jul., 2003.

[14] H. Luo, R. Ramjee, P. Sinha, L. Li, and S. Lu, "Cellular and hybrid networks: UCAN: a unified cellular and Ad-Hoc network architecture", ACM Proceedings conference on Mobile computing and networking, Sep., 2003.

[15] C. S. Wijting, and R. Prasad, "Evaluation of mobile ad-hoc network techniques in a cellular network", IEEE Conference on Vehicular Technology, vol.3, pp. 1025-1029, Sep., 2000.

[16] H. Wu, C. Qiao, S. De, and O. Tonguz, "Integrated Cellular and Ad Hoc Relaying Systems: iCAR.”, IEEE Journal on Selected Areas in Communications, vol.19, no.10, pp.2105-2115, Oct., 2001

[17] E. Karamad and F. Ashtiani, "A modified 802.11based MAC scheme to assure fair access for vehicle-to-roadside communications," Computer communications, Vol. 31, 2008

[18] J. Broch, D. A. Maltz, and D. B. Johnson, "Supporting hierarchy and heterogeneous interfaces in multi-hop wireless ad hoc networks", (I-SPAN) Proceedings on Parallel Architectures, Algorithms, and Networks, pp.370-375, Jun.,1999.

[19] Chia- Chen, H., H. Chan, et al. 2008, Mobility Pattern Aware Routing for Heterogeneous Vehicular Networks. IEEE Wireless Communications and Networking Conference, WCNC 2008 
[20] Choffnes, D. R., Fabin, et al. 2005, An integrated mobility and traffic model for vehicular wireless networks, Proceedings of the 2nd ACM international workshop on Vehicular ad hoc networks, Cologne, Germany, ACM.

[21] E. Karamad and F. Ashtiani, "A modified 802.11based MAC scheme to assure fair access for vehicle-to-roadside communications," Computer communications, Vol. 31, 2008

[22] A. Mahajan,'Evaluation of Mobility Models for Vehicular Ad-hoc Network Simulations", TechnicalReportN.051220, Florida State University, 2005.

[23] Babak Pazand, Chris McDonald," A Critique of Mobility Models for Wireless Network Simulation", University of Western Australia, 6th IEEE/ACS International Conference on Computer and Information Science (ICIS 2007).

[24] Biswas, S., R. Tatchikou, et al. 2006, "Vehicle-to-vehicle wireless communication protocols for enhancing highway traffic safety," Communications Magazine, IEEE 44(1): 74-82. 